\section{Maternal morbidity and perinatal outcomes in rural versus urban areas}

We commend Lisonkova and colleagues for the breadth of morbidities they included in their article. ${ }^{1}$ However, we are disappointed and somewhat astonished over the absence of any attempt to include an analysis of a crucial variable in the discussion: distance to services. This limitation not only weakens the conclusions of the study, but also calls into question the validity of the findings.

The authors note, "The limitations of our study include the lack of individual information on the time needed to travel to the nearest health care facility...." Although individual information would be ideal, all we need to know is whether birthing women have access to maternity services in their community. This lack of service-level consideration undermines the article. There is strong evidence from British Columbia and internationally that local access to maternity care is an important influence on maternal newborn outcomes.

This lack of attention to distance creates a conceptual shortcoming: the rural group has been defined by its isolation from population centres (i.e., maternity health services), but distance to services (predictor of outcomes) is ignored in the cohort analysis. From previous work with an overlapping data set, we predict that of the 25855 rural cases, between 4000 and 6000 will be from communities that are more than one hour from the nearest maternity services. This could easily account for the relatively minor differences in the odds ratios for the three principal morbidities (eclampsia, obstetric embolism and uterine scar dehiscence/rupture).

Once distance to services is accounted for, data from $\mathrm{BC}$ and Canada show that women from communities without maternity services have poorer outcomes than those from com- munities with services. Data also show that women from communities with primary maternity care (i.e., no cesarean delivery) and communities where cesarean delivery is provided by family physicians with enhanced surgical skills have outcomes as good as those from communities with obstetricians providing care. To suggest, as the authors do in their conclusion, that in rural communities "the emphasis should remain on monitoring" for those conditions "requiring advanced obstetric and neonatal care" is not only misguided, but also impugns the excellent maternity services being provided in communities that are still offering services.

We question why CMAJ published this manuscript. It is a weak cohort analysis that ignores the key health services determinants of outcomes for rural maternity care, but then makes recommendations about the organization of health services. The article presents misleading and potentially frightening data for women in rural areas who are trying to decide where they should give birth. A worthwhile adjustment to the analysis of this data would be to stratify those women according to whether they have a local maternity service in their community, and then examine morbidities. The literature has already demonstrated good outcomes for newborns. We expect that data will show the same for maternal outcomes.

\section{Stefan Grzybowski MD}

Jude Kornelsen PhD

Centre for Rural Health Research,

Department of Family Practice, University of British Columbia, Vancouver, BC

\section{Reference}

1. Lisonkova S, Haslam MD, Dahlgren L, et al. Maternal morbidity and perinatal outcomes among women in rural versus urban areas. CMAJ 2016;188:E456-65.

\section{CMAJ 2016. DOI:10.1503/cmaj.1150130}

On behalf of the Society of Rural Physicians of Canada, we believe that the study by Lisonkova and col- leagues ${ }^{1}$ does a disservice to rural maternity providers, and fails to address the factors that most influence maternal morbidity and perinatal outcomes.

The authors allude to the fact that closure of rural maternity units may have played a role in the outcomes in their study; however, they downplay this fact and choose to focus on the providers, suggesting that, "the emphasis should remain on monitoring for potentially life-threatening maternal and perinatal complications...." In our experience, rural maternity care practitioners would not neglect to notice when preeclampsia progressed to eclampsia, for example. With reduced access to maternity care, women in rural Canada will present later, attend less frequent appointments or even choose to avoid transfer for delivery, which results in an increased risk of complications. Further, general health care teams are less prepared than rural teams when women make these choices.

We cannot ignore the need to provide local access to care. Pregnant women in rural areas tend to be younger, have higher rates of smoking or substance use, and have preexisting hypertension. When a pregnancy is labelled high risk, will a woman choose to leave her family, sometimes for weeks before delivery, and travel hundreds (or thousands) of kilometres to receive care? Will she want to deliver where the culture and language may be different, at substantial personal financial cost, and where her support people may not be present?

In areas with primary maternity care (no cesarean delivery), lowvolume maternity units or maternity care with family physicians with enhanced surgical skills for cesarean delivery, rural women have outcomes equal to those of their urban counterparts. As rural and urban maternity care providers, we should be advocating strongly for the strengthening of rural maternity services to improve 